\title{
Models for the association between ordinal variables
}

\author{
Krista Lapp ${ }^{\mathrm{a}}$, Geert Molenberghs ${ }^{\mathrm{b}, *}$, Emmanuel Lesaffre ${ }^{\mathrm{c}}$ \\ ${ }^{\text {a } U n i v e r s i t y ~ o f ~ E s t o n i a, ~ T a r t u, ~ E s t o n i a ~}$ \\ ${ }^{\mathrm{b}}$ Limburgs Universitaira Centrum, Universitaire Campus, Building D, B-3590 Diepenbeek, Belgium \\ ${ }^{\mathrm{C}}$ Katholieke Universiteit Leuven, Leuven, Belgium
}

Received 1 February 1997; received in revised form 1 December 1997; accepted 2 April 1998

\begin{abstract}
In this paper we present marginal association and regression models as an alternative to classical association models for cross-classified ordinal data. It is shown that the methods easily incorporate various types of association structures, are able to include covariate information and generalize easily to multi-way classifications. The proposed approach is used to analyze data from a multicentre psychiatric study. (C) 1998 Elsevier Science B.V. All rights reserved.
\end{abstract}

Keywords: Cross-ratio model; Marginal association model; Marginal regression model; Plackett distribution; RC model; Uniform association model

\section{Introduction}

Over the past decades, a vast part of the literature, devoted to multivariate categorical data, focused on describing the association structure between two or more variables. Eminent references are Yule and Kendall (1950) and Goodman (1969, $1979,1981 \mathrm{a}, \mathrm{b}, 1983$ ). A commonly used measure of association is the cross-ratio.

Recently, the focus in multivariate categorical data has somewhat shifted to regression models, intended mainly for the analysis of longitudinal data. A wide class is provided by the so-called marginal models. Non-likelihood marginal methods, such as generalized estimating equations (Liang and Zeger, 1986) and likelihood based marginal methods (Dale, 1986; Molenberghs and Lesaffre, 1994; Lang and Agresti, 1994; Glonek and McCullagh, 1995) have been proposed. It is now common practice to describe the association between repeated binary outcomes by two-way and

\footnotetext{
${ }^{*}$ Corresponding author. Tel.: (+32 11) 26 8238; fax: (+32 11) 26 8299; e-mail: gmolenb@luc.ac.be.
} 
Table 1

Cross-classification of British male sample according to each subject's occupational status category and his father's occupational category, using seven status categories

\begin{tabular}{|c|c|c|c|c|c|c|c|}
\hline \multirow{2}{*}{$\begin{array}{l}\text { Father's } \\
\text { status }\end{array}$} & \multicolumn{7}{|c|}{ Subject's status } \\
\hline & 1 & 2 & 3 & 4 & 5 & 6 & 7 \\
\hline 1 & 50 & 19 & 26 & 8 & 18 & 6 & 2 \\
\hline 2 & 16 & 40 & 34 & 18 & 31 & 8 & 3 \\
\hline 3 & 12 & 35 & 65 & 66 & 123 & 23 & 21 \\
\hline 4 & 11 & 20 & 58 & 110 & 223 & 64 & 32 \\
\hline 5 & 14 & 36 & 114 & 185 & 714 & 258 & 189 \\
\hline 6 & 0 & 6 & 19 & 40 & 179 & 143 & 71 \\
\hline 7 & 0 & 3 & 14 & 32 & 141 & 91 & 106 \\
\hline
\end{tabular}

higher-order cross-ratios. Molenberghs and Lesaffre (1994) developed their method for multivariate ordinal data. For this kind of data, they advocate the use of the global cross-ratio. This extends the work by Dale, who exploited the structure of the bivariate Plackett distribution to estimate the parameters in a global cross-ratio model. Molenberghs and Lesaffre (1997b) generalize this method to a wide class of association measures, applicable to both ordinal and nominal data. However, it is not sufficiently recognized that these models provide a versatile basis, not only for regressing multiple outcomes on predictors, but also to study the association between two (or more) categorical variables. In other words, they can be used for the analysis of association. To this end, it is necessary to construct more complex association models than is customary in the marginal modelling framework. For this purpose, one can borrow flexible models such as described in Goodman (1981a).

Two examples are presented. First, to illustrate the use of the models in the case of contingency tables with a large number of categories, we re-analyze the data presented in Goodman (1979). Subjects are cross-classified, according to their occupational status and their father's occupational status, using seven ordered categories. The data are presented in Table 1.

The main example is a multicentre study involving 315 patients that were treated by fluvoxamine for psychiatric symptoms described as possibly resulting from a dysregulation of serotonine in the brain. Patients with one or more of the following diagnoses were included: depression, obsessive, compulsive disorder and panic disorder. Several covariates were recorded, such as sex and initial severity on a 5point ordinal scale, where severity increases with category. After recruitment of the patient in the study, he or she was investigated at three visits. On the basis of about 20 psychiatric symptoms, the therapeutic effect and the side-effects were scored at each visit in an ordinal manner. Side effect is coded as $(1)=$ no; $(2)=$ not interfering with functionality of patient; $(3)=$ interfering significantly with functionality of patient; (4) = the side-effect surpasses the therapeutic effect. Similarly, the effect of therapy is recorded on a four-point ordinal scale: (1) no improvement over baseline or worsening; (2) minimal improvement (not changing functionality); (3) moderate improvement (partial disappearance of symptoms) and (4) important improvement 
Table 2

Psychiatric study: cross-classification of initial severity and side effect at the second occasion. The fitted values for the independence model are shown in parentheses

\begin{tabular}{lcccc}
\hline Severity & \multicolumn{4}{c}{ Side 2} \\
\cline { 2 - 5 } & 1 & 2 & 3 & 4 \\
\hline 1 & 1 & 0 & 1 & 0 \\
& $(0.86)$ & $(0.86)$ & $(0.18)$ & $(0.10)$ \\
2 & 21 & 28 & 5 & 5 \\
& $(25.29)$ & $(25.29)$ & $(5.42)$ & $(3.01)$ \\
3 & 62 & 62 & 15 & 7 \\
& $(62.57)$ & $(62.57)$ & $(13.41)$ & $(7.45)$ \\
4 & 41 & 31 & 6 & 2 \\
& $(34.29)$ & $(34.29)$ & $(7.35)$ & $(4.08)$ \\
5 & 1 & 5 & 0 & 1 \\
& $(3.00)$ & $(3.00)$ & $(0.64)$ & $(0.36)$ \\
\hline
\end{tabular}

(almost disappearance of symptoms). Thus, a side effect occurs if new symptoms occur while there is therapeutic effect if old symptoms disappear.

To illustrate the flexibility of the proposed approach, we select four two-way classifications from the psychiatric study. We will first consider a cross-classification of side effects and initial severity (Table 2 ). Then, we cross-classify the measurements on therapeutic effect at visits 2 and 3 in Table 3. A similar table is constructed for side effects (Table 4). Finally, we consider a cross-classification of side effects and therapeutic effect, recorded at visit 2 (Table 5).

Note that the total of Table 5 (299) is higher than the total of Table 2 (294), since there are five subjects with information on therapeutic and side effects, but without initial severity measurement.

These tables cover different settings: a cross-classification of an outcome and a baseline variable, the same outcome at subsequent measurement times and a "crosssectional" picture, comprised of two variables measured simultaneously.

In Section 2, we first sketch the so-called multivariate logistic models (McCullagh and Nelder, 1989; Glonek and McCullagh, 1995). Then we review the classical RC association models (Goodman, 1981a) and the marginal association model (Dale, 1986; Molenberghs and Lesaffre, 1994). It is indicated that both families can be seen as specific multivariate logistic models. This observation naturally leads to the observation that within the multivariate logistic models family, very general association models can be constructed. Tables 1-5 are analyzed in Section 3. The psychiatric study calls for extension of the methodology beyond a single twoway classification. This problem is addressed in Section 4. First, it is shown how covariates can be included. E.g., if a cross-classification is made for males and females separately, the sex effect in both the marginal responses and the interactions can be studied. Also, continuous covariates such as age can be incorporated. Further, the methodology is not restricted to two-way classifications, as arbitrary cross-classifications can be handled as well. Several proposals for three- or higher- 
Table 3

Psychiatric study: cross-classification of therapeutic effect at the second and third occasion. The fitted values are in parentheses: the first entry corresponds to the constant association Dale model, while the second entry stands for the row and column local association model

\begin{tabular}{lcccc}
\hline Ther. 2 & \multicolumn{4}{c}{ Ther. 3 } \\
\cline { 2 - 5 } & 1 & 2 & 3 & 4 \\
\hline 1 & 13 & 2 & 0 & 0 \\
& $(11.64)$ & $(2.87)$ & $(0.49)$ & $(0.15)$ \\
& $(13.06)$ & $(1.87)$ & $(0.06)$ & $(0.01)$ \\
2 & 37 & 40 & 8 & 4 \\
& $(40.46)$ & $(39.77)$ & $(5.50)$ & $(1.39)$ \\
& $(34.98)$ & $(44.28)$ & $(7.55)$ & $(2.20)$ \\
3 & 13 & 58 & 18 & 4 \\
& $(10.09)$ & $(53.94)$ & $(23.38)$ & $(4.77)$ \\
& $(15.65)$ & $(52.42)$ & $(18.49)$ & $(6.45)$ \\
4 & 1 & 13 & 36 & 21 \\
& $(2.68)$ & $(16.71)$ & $(32.52)$ & $(21.64)$ \\
& $(0.32)$ & $(14.44)$ & $(35.91)$ & $(20.34)$ \\
\hline
\end{tabular}

Table 4

Psychiatric study: cross-classification of side effects at the second and third occasion. The fitted values are in parentheses: the first entry corresponds to the row and column effects Dale model, while the second entry corresponds to model (2.6)

\begin{tabular}{llccc}
\hline Side 2 & \multicolumn{5}{c}{ Side 3} \\
\cline { 2 - 5 } & 1 & 2 & 3 & 4 \\
\hline 1 & 105 & 14 & 0 & 0 \\
& $(104.98)$ & $(13.84)$ & $(0.16)$ & $(0.00)$ \\
& $(105.01)$ & $(13.98)$ & $(0.01)$ & $(0.00)$ \\
2 & 34 & 80 & 7 & 1 \\
& $(33.88)$ & $(80.46)$ & $(7.27)$ & $(0.27)$ \\
& $(33.63)$ & $(79.96)$ & $(8.20)$ & $(0.22)$ \\
3 & 2 & 7 & 10 & 2 \\
& $(2.09)$ & $(7.02)$ & $(8.76)$ & $(2.91)$ \\
& $(2.71)$ & $(7.14)$ & $(7.58)$ & $(3.57)$ \\
4 & 3 & 1 & 0 & 2 \\
& $(3.14)$ & $(1.01)$ & $(0.00)$ & $(2.21)$ \\
& $(2.65)$ & $(0.92)$ & $(1.21)$ & $(1.22)$ \\
& & & &
\end{tabular}

dimensional tables are discussed, based on generalizing Dale's model and the RC model. A cross-classification of the therapeutic effect at measurement occasions 2, 3, and 4 is used to illustrate that the marginal regression family is able to summarize this kind of three-way tables in a fairly parsimonious way. Support to this claim is given using a few other cross-classifications from the psychiatric study.

In Section 5 it is indicated how the association models can be embedded in families of models, arising as discretizations of continuous distributions. A commonly 
Table 5

Psychiatric study: cross-classification of side effects and therapeutic effect at the second occasion. The fitted values are shown are in parentheses. The first model is the global association column effects model. The second global cross-ratio model includes row and column effects, as well as interactions. The third set of fitted values corresponds to the $\mathrm{RC}$ model (row and column effects)

\begin{tabular}{|c|c|c|c|c|}
\hline \multirow[t]{2}{*}{ Side 2} & \multicolumn{4}{|c|}{ Therapeutic 2} \\
\hline & 1 & 2 & 3 & 4 \\
\hline \multirow[t]{4}{*}{1} & 8 & 40 & 40 & 40 \\
\hline & $(7.46)$ & $(38.32)$ & $(44.19)$ & $(38.11)$ \\
\hline & $(8.10)$ & $(39.58)$ & $(40.41)$ & $(39.50)$ \\
\hline & $(8.91)$ & $(38.18)$ & $(41.85)$ & $(39.05)$ \\
\hline \multirow[t]{4}{*}{2} & 7 & 45 & 51 & 25 \\
\hline & $(9.73)$ & $(45.60)$ & $(43.33)$ & $(29.09)$ \\
\hline & $(6.61)$ & $(46.37)$ & $(49.46)$ & $(25.49)$ \\
\hline & $(6.33)$ & $(46.92)$ & $(48.99)$ & $(25.75)$ \\
\hline \multirow[t]{4}{*}{3} & 2 & 9 & 8 & 9 \\
\hline & $(1.37)$ & $(7.82)$ & $(10.39)$ & $(8.52)$ \\
\hline & $(2.22)$ & $(7.49)$ & $(9.66)$ & $(8.64)$ \\
\hline & $(2.02)$ & $(8.12)$ & $(8.95)$ & $(8.91)$ \\
\hline \multirow[t]{5}{*}{4} & 2 & 1 & 3 & 9 \\
\hline & $(0.32)$ & $(2.15)$ & $(4.45)$ & $(8.15)$ \\
\hline & $(2.24)$ & $(1.30)$ & $(2.52)$ & $(8.95)$ \\
\hline & $(1.74)$ & $(1.77)$ & $(2.20)$ & $(9.29)$ \\
\hline & \multicolumn{4}{|c|}{ Male subjects } \\
\hline 1 & 4 & 18 & 12 & 16 \\
\hline 2 & 0 & 9 & 19 & 9 \\
\hline 3 & 0 & 4 & 3 & 4 \\
\hline 4 & 0 & 1 & 1 & 5 \\
\hline \multicolumn{5}{|c|}{ Female subjects } \\
\hline 1 & 4 & 22 & 28 & 24 \\
\hline 2 & 7 & 36 & 32 & 16 \\
\hline 3 & 2 & 5 & 5 & 5 \\
\hline 4 & 2 & 0 & 2 & 4 \\
\hline
\end{tabular}

used argument in favour of RC models is their well-known capability to approximate discretizations of the bivariate normal distribution. The model used in this context is Goodman's uniform association model (Goodman, 1981a). Elements to this discussion are brought forward by Goodman (1981b, 1983) and Becker (1989). We indicate that this relationship naturally follows from the results of Holland and Wang (1987) on the local dependence function (LDF). Also, a similar correspondence holds between the marginal global cross-ratio models and the Plackett distribution. We argue that the choice between different models should not be made on the ground of potential classes of underlying densities, but on the structure of associations. The fact that the different models treated here are multivariate logistic models gives additional support to this point of view. 


\section{Association models}

We first introduce the necessary notation. Suppose a contingency table arises from cross-classifying $n$ subjects w.r.t. two categorical variables $Y_{1}$ and $Y_{2}$, having $I$ and $J$ levels, respectively. It is convenient to introduce both ordinary multinomial cell counts

$$
Z_{i j r}^{*}=\left\{\begin{array}{l}
1 \text { if } y_{1 r}=i \text { and } y_{2 r}=j \\
0 \text { otherwise, }
\end{array}\right.
$$

as well as their cumulative counterparts

$$
Z_{i j r}= \begin{cases}1 & \text { if } y_{1 r} \leq i \text { and } y_{2 r} \leq j \\ 0 & \text { otherwise }\end{cases}
$$

with a subscript $r$ denoting the $r$ th subject. The corresponding probabilities are defined by $\mu_{i j}^{*}=\operatorname{pr}\left(Z_{i j r}^{*}=1\right)$ and $\mu_{i j}=\operatorname{pr}\left(Z_{i j r}=1\right)$. This notation will be used to describe the association models. Should the probabilities depend on the subject (for example through the introduction of covariate information), then a subscript $r$ will be added $\left(\mu_{i j r}^{*}\right.$ and $\left.\mu_{i j r}\right)$. We will first introduce a general framework, largely due to McCullagh and Nelder (1989) and Glonek and McCullagh (1995). Then, the RC family of models (Goodman, 1981a) and the Dale (1986) model are shown to fit within this framework, conditional on a slight generalization in the RC case. Finally, it is indicated how the modelling framework can be used to combine useful aspects of both subclasses, to yield a very wide and versatile class which in addition allows extension to covariates as well as to higher-order tables.

\subsection{Multivariate logistic models}

McCullagh and Nelder (1989) defined a useful class of generalized linear models, by writing the vector link function in terms of the joint probabilities in the following way:

$$
\eta=C^{\mathrm{T}} \ln \left(L \mu^{*}\right)
$$

with $\mu^{*}$ the vector of joint probabilities, formed by stacking the $\mu_{i j}^{*}$. The matrix $L$ consists solely of zeros and ones, such that $L \mu^{*}$ contains the probabilities necessary to construct the required link functions. Then, contrasts of log-probabilities are equated to a vector of linear predictors $\eta$ using the contrast matrix $C$. Contrasts of logprobabilities encompass many commonly used links for both marginal probabilities and associations. Within this model formulation, the marginal means can be modelled via, e.g. baseline-category logits, adjacent category logits, continuation-ratio logits, or cumulative logits. The association can be described in terms of, e.g. local or global cross-ratios. This means that this formulation applies to binary, ordinal, and nominal data. When cumulative logits and/or global cross-ratios are used, the model can be expressed directly in terms of the cumulative probabilities $\mu_{i j}$, such that (2.1) becomes $\boldsymbol{\eta}=C^{\mathrm{T}} \ln (L \mu)$. In this case, $L$ may contain other elements than merely 
zeros and ones. Alternatively, the connection between $\mu_{i j}$ and $\mu_{i j}^{*}\left(\mu=B \mu^{*}\right.$, for some constant matrix $B$ ) can be absorbed into the matrix $L$ as well. As counterexamples, modelling the marginal distribution via, e.g. the probit or the complementary log$\log$ link is excluded from (2.1). One usually requires that $\mu^{*}$ and $\eta$ are in 1-to-1 relationship. Model (2.1) is called the multivariate logistic transform by Glonek and McCullagh (1995). They illustrate its use for both marginal and conditional regression models, as well as for mixed marginal-conditional parameterizations. A general and flexible class of marginal logistic models of the form (2.1) was studied by Lang and Agresti (1994), who allow a many-to-one relationship between $\boldsymbol{\mu}^{*}$ and $\boldsymbol{\eta}$ since they do not require that the (higher-order) associations are modelled explicitly. Examples will be given in the next two sections.

In the spirit of generalized linear modelling, McCullagh and Nelder (1989) completed (2.1) by

$$
\eta=X \xi
$$

i.e., by adopting a vector of linear predictors. Here, $X$ is a known design and/or covariate matrix and $\xi$ is a vector of parameters of direct interest. Glonek and McCullagh (1995) call the resulting family multivariate logistic regression models.

When not only regression aspects are of scientific interest, but focus is placed on the association structure as well, it is useful to generalize the vector of linear predictors $(2.2)$ to the potentially non-linear class

$$
\boldsymbol{\eta}=C^{\mathrm{T}} \ln \left(L \boldsymbol{\mu}^{*}\right)=\boldsymbol{g}(\boldsymbol{\xi})
$$

where $\boldsymbol{g}(\xi)$ is a known vector-valued function.

\subsection{Goodman's local association models}

Goodman (1981a) defines association models in terms of log local cross-ratios for $I \times J$ tables. These log cross-ratios are given by

$$
\ln \theta_{i j}^{*}=\ln \left(\frac{\operatorname{pr}\left(Y_{1}=i, Y_{2}=j\right) \operatorname{pr}\left(Y_{1}=i+1, Y_{2}=j+1\right)}{\operatorname{pr}\left(Y_{1}=i, Y_{2}=j+1\right) \operatorname{pr}\left(Y_{1}=i+1, Y_{2}=j\right)}\right)=\ln \frac{\mu_{i j}^{*} \mu_{i+1, j+1}^{*}}{\mu_{i, j+1}^{*} \mu_{i+1, j}^{*}},
$$

with $i=1, \ldots, I-1$ and $j=1, \ldots, J-1$. They naturally follow from the following closed-form model for the joint cell probabilities:

$$
\mu_{i j}^{*}=\alpha_{i} \beta_{j} \mathrm{e}^{\phi \lambda_{i} v_{j}}
$$

$(i=1, \ldots, I ; j=1, \ldots, J)$. Here, $\alpha_{i}$ and $\beta_{j}$ are main effect parameters while $\lambda_{i}, v_{j}$ and $\phi$ describe the association structure. Indeed, the local cross-ratios are $\ln \theta_{i j}^{*}=$ $\phi\left(\lambda_{i}-\lambda_{i+1}\right)\left(v_{j}-v_{j+1}\right)$. Identifiability constraints have to be imposed on the parameters in (2.4). This model is also called the row-column model ( $\mathrm{RC}$ model).

Model (2.4) can be seen as a member of (2.3) by setting $L$ and $C$ equal to the identity matrix: $\boldsymbol{\eta}=\ln \mu^{*}=g(\xi)$, with $g(\xi)$ defined by

$$
g_{i j}(\xi)=\ln \alpha_{i}+\ln \beta_{j}+\phi \lambda_{i} v_{j}
$$


Due to its third term, the predictor function (2.5) is non-linear. Note that (2.5) is a mixture of main effect and association parameters. By setting $C$ equal to the identity matrix, the concept of contrasts of log-probabilities is not maintained and thus (2.3) is slightly extended.

An alternative association parameterization is additive in the log cross-ratios: In $\theta_{i j}^{*}=\delta_{1 i}+\delta_{2 j}$. This model is induced by the following expression for the cell probabilities:

$$
\mu_{i j}^{*}=\alpha_{i} \beta_{j} \gamma_{1 i}^{j} \gamma_{2 j}^{i}
$$

For this parameterization (2.5) changes to

$$
g_{i j}(\xi)=\ln \alpha_{i}+\ln \beta_{j}+j \ln \gamma_{1 i}+i \ln \gamma_{2 j} .
$$

Note that this predictor is of the linear type in $\ln \alpha_{i}$, etc. Fitting algorithms for (2.4) and (2.6) can be found in Goodman (1981a).

Goodman (1981a) generalizes model $(2.4)$ to

$$
\mu_{i j}^{*}=\alpha_{i} \beta_{j} \exp \left(\sum_{k=1}^{4} \phi_{k} \lambda_{k i} v_{k j}\right),
$$

where $\lambda_{1 i}$ and $\lambda_{3 i}$ are linear functions of the index $i$ and $v_{1 j}$ and $v_{2 j}$ are linear in $j$. The others are allowed to be non-linear. He shows that the log cross-ratios can be written as

$$
\ln \theta_{i j}^{*}=\eta+\eta_{i}^{I}+\eta_{j}^{J}+\zeta_{i}^{I} \zeta_{j}^{J} .
$$

This models allows the inclusion of additive effects on the association. Goodman calls it the $\mathrm{R}+\mathrm{C}+\mathrm{RC}$ model.

Although the above models provide an elegant description of the association in contingency tables, a disadvantage of the RC family is the cumbersome form they induce for the marginal distributions. The model presented next is built from the marginal probabilities.

\subsection{Dale's marginal models}

Dale (1986) and Molenberghs and Lesaffre (1994, 1997b) define a marginal model for ordinal data in terms of marginal cumulative logits and global cross-ratios. The cumulative logits

$$
\begin{aligned}
& \eta_{1 i}=\operatorname{logit}\left(\operatorname{pr}\left(Y_{1} \leq i\right)\right)=\ln \left(\mu_{i J}\right)-\ln \left(1-\mu_{i J}\right), \\
& \eta_{2 j}=\operatorname{logit}\left(\operatorname{pr}\left(Y_{2} \leq j\right)\right)=\ln \left(\mu_{I j}\right)-\ln \left(1-\mu_{I j}\right),
\end{aligned}
$$

$(i=1, \ldots, I-1 ; j=1, \ldots, J-1)$, and the global cross-ratios

$$
\begin{aligned}
\ln \psi_{i j} & =\ln \left(\frac{\operatorname{pr}\left(Y_{1} \leq i, Y_{2} \leq j\right) \operatorname{pr}\left(Y_{1}>i, Y_{2}>j\right)}{\operatorname{pr}\left(Y_{1} \leq i, Y_{2}>j\right) \operatorname{pr}\left(Y_{1}>i, Y_{2} \leq j\right)}\right) \\
& =\ln \frac{\mu_{i j}\left(1-\mu_{I j}-\mu_{i J}+\mu_{i j}\right)}{\left(\mu_{i J}-\mu_{i j}\right)\left(\mu_{I j}-\mu_{i j}\right)}
\end{aligned}
$$

define the joint probabilities. 
It is clear from (2.10)-(2.12) that this model is a member of $(2.1)$. For the special case of binary data $(I=J=2),(2.1)$ becomes

$$
\left(\begin{array}{l}
\eta_{1} \\
\eta_{2} \\
\eta_{3}
\end{array}\right)=\left(\begin{array}{rrrrrrrr}
0 & 0 & 0 & 0 & 1 & -1 & 0 & 0 \\
0 & 0 & 0 & 0 & 0 & 0 & 1 & -1 \\
1 & -1 & -1 & 1 & 0 & 0 & 0 & 0
\end{array}\right) \ln \left(\begin{array}{llll}
1 & 0 & 0 & 0 \\
0 & 1 & 0 & 0 \\
0 & 0 & 1 & 0 \\
0 & 0 & 0 & 1 \\
1 & 1 & 0 & 0 \\
0 & 0 & 1 & 1 \\
1 & 0 & 1 & 0 \\
0 & 1 & 0 & 1
\end{array}\right)\left(\begin{array}{c}
\mu_{11}^{*} \\
\mu_{12}^{*} \\
\mu_{21}^{*} \\
\mu_{22}^{*}
\end{array}\right)
$$

where the model is written in terms of the cell probabilities $\mu_{j k}^{*}$. Since

$$
\left(\begin{array}{l}
\mu_{11}^{*} \\
\mu_{12}^{*} \\
\mu_{21}^{*} \\
\mu_{22}^{*}
\end{array}\right)=\left(\begin{array}{rrrr}
1 & 0 & 0 & 0 \\
-1 & 1 & 0 & 0 \\
-1 & 0 & 1 & 0 \\
1 & -1 & -1 & 1
\end{array}\right)\left(\begin{array}{l}
\mu_{11} \\
\mu_{12} \\
\mu_{21} \\
\mu_{22}
\end{array}\right)
$$

it is clear that an cxpression in tcrms of the cumulative probabilities $\mu_{j k}$ is immediatc.

Should it be thought reasonable, then local cross-ratios:

$$
\ln \psi_{i j}^{*}=\ln \frac{\mu_{i j}^{*}\left(1-\mu_{i+1, j}^{*}-\mu_{i, j+1}^{*}+\mu_{i j}^{*}\right)}{\left(\mu_{i, j+1}^{*}-\mu_{i j}^{*}\right)\left(\mu_{i+1, j}^{*}-\mu_{i j}^{*}\right)}
$$

can be used instead. For the particular case of binary variables, both types of crossratios coincide.

For the association (2.12), we will pay particular attention to

$$
\ln \psi_{i j}=\phi+\rho_{1 i}+\rho_{2 j}+\sigma_{1 i} \sigma_{2 j}
$$

including a constant association parameter, row and column effects, and interactions between rows and columns, respectively. This model is identified, e.g. by imposing $\rho_{1 I}=\rho_{2 J}=\sigma_{1 I}=\sigma_{2 J}=0$ and $\sigma_{11}=1$. Due to the fourth term of (2.14) this parameterization is a member of the non-linear family (2.3). It is very similar in structure to the local cross-ratios of the $\mathrm{R}+\mathrm{C}+\mathrm{RC}$ model (2.9). Of course, model (2.14) is only one of many possibilities, since there is a whole spectrum of possible models between independence and constant association on the one hand and a saturated association model on the other hand. When the number of categories increases, it becomes more crucial to look for parsimonious association models in order to reduce the number of parameters in the model. To this end, the more flexible class (2.3) might be preferable over (2.2).

Model fitting proceeds, e.g. via Newton-Raphson or Fisher scoring techniques. In order to do so, the cumulative cell probabilities need to be computed. First, note that $\mu_{I J}=1$. Then, $\mu_{i J}$ and $\mu_{I j}$ follow from $\eta_{1 i}$ and $\eta_{2 j}$, i.e. Eqs. (2.10) and (2.11) 
are solved for $\mu_{i J}$ and $\mu_{l j}$. The other counts follow from

$$
\mu_{i j}= \begin{cases}\frac{1+\left[\mu_{i J}+\mu_{I j}\right]\left(\psi_{i j}-1\right)-S_{i j}}{2\left(\psi_{i j}-1\right)} & \text { if } \psi_{i j} \neq 1, \\ \mu_{i J} \mu_{I j} & \text { otherwise, }\end{cases}
$$

where

$$
S_{i j}=\sqrt{\left[1+\left(\psi_{i j}-1\right)\left(\mu_{i J}+\mu_{i j}\right)\right]^{2}+4 \psi_{i j}\left(1-\psi_{i j}\right) \mu_{i J} \mu_{I j}} .
$$

Molenberghs and Lesaffre $(1994,1997 \mathrm{~b})$ show how to extend this class of models to more than two variables. They also indicate how to adopt other association measures, such as marginal correlations, which corresponds to the Bahadur (1961) model. Molenberghs (1994) and Lesaffre et al. (1994) provide details on maximum likelihood estimation for the two-way and higher-order versions of the model.

\subsection{A general class of models}

The models described in Sections 2.2 and 2.3 differ in two respects:

1. The association in the RC model is in terms of local cross-ratios, while the Dale model is based on global cross-ratios. This difference is not essential, since we argued that local cross-ratios can be incorporated in the marginal model without problem.

2. The marginal probabilities of the $\mathrm{RC}$ model are complex functions of the model parameters, whereas the Dale model is expressed directly in terms of the marginal logits.

However, upon generalizing (2.3) slightly, both models are seen as subclasses of this flexible family. For both models, linear and non-linear predictors are possible. Indeed, for the RC family, (2.7) is linear whereas (2.5) is non-linear. For the Dale model, (2.14) is non-linear, but if the fourth term is dropped, it becomes a linear predictor.

The advantage of this result is that completely general models can be constructed, combining and extending interesting aspects of both the RC and the Dale model. For example, a genuine marginal model can be constructed, with an association function of the RC type. Depending on the data problem, one can opt for local or for global cross-ratios. Arguably, local cross-ratios are suitable for nominal variables, whereas global cross-ratios are a natural choice for cross-classified ordinal variables.

\section{Analysis of examples}

Standard RC and Dale models, fitted to Table 1, are presented in Table 6. The Dale model with row effects, column effects, and interactions, provides a good fit. This means that no model of the form (2.2) fits the data and that the full non-linear version (2.14) is necessary to achieve a acceptable fit. No RC model, not even the $\mathrm{R}+\mathrm{C}+\mathrm{RC}$ model, fits the data well. 
Table 6

Deviance $\chi^{2}$ goodness-of-fit statistics for Dale and RC Models, Fitted to the data in Table 1 . The models with an acceptable fit are indicated by an asterisk

\begin{tabular}{|c|c|c|c|c|}
\hline \multirow[t]{2}{*}{ Description } & \multicolumn{2}{|c|}{ Dale } & \multicolumn{2}{|c|}{$\mathrm{RC}$} \\
\hline & $\mathrm{df}$ & $\chi^{2}$ & $\mathrm{df}$ & $\chi^{2}$ \\
\hline Independence & 36 & 897.52 & 36 & 897.52 \\
\hline Constant association & 35 & 207.23 & 35 & 98.19 \\
\hline Row effects only & 30 & 105.23 & 30 & 87.14 \\
\hline Column effects only & 30 & 100.69 & 30 & 80.74 \\
\hline Row and column effects & 25 & 42.94 & 25 & 75.59 \\
\hline Row, column, interactions & 16 & ${ }^{*} 20.11$ & 16 & 38.09 \\
\hline Saturated model & 0 & 0.00 & 0 & 0.00 \\
\hline
\end{tabular}

Table 7

Deviance $\chi^{2}$ goodness-of-fit statistics for Dale and RC models, fitted to Tables 2-5 (models with an acceptable fit are indicated by an asterisk)

\begin{tabular}{|c|c|c|c|c|c|c|c|c|}
\hline \multirow[t]{2}{*}{ Description } & \multicolumn{2}{|c|}{ Table 2} & \multicolumn{2}{|c|}{ Table 3} & \multicolumn{2}{|c|}{ Table 4} & \multicolumn{2}{|c|}{ Table 5} \\
\hline & df & $\chi^{2}$ & df & $\chi^{2}$ & df & $\chi^{2}$ & $\mathrm{df}$ & $\chi^{2}$ \\
\hline \multicolumn{9}{|l|}{ Dale models } \\
\hline Independence & 12 & ${ }^{*} 14.20$ & 9 & 141.95 & 9 & 158.15 & 9 & 17.12 \\
\hline Constant association & 11 & ${ }^{*} 11.71$ & 8 & ${ }^{*} 11.48$ & 8 & 18.27 & 8 & 17.12 \\
\hline Row effects only & 8 & ${ }^{*} 8.34$ & 6 & ${ }^{*} 3.80$ & 6 & 14.49 & 6 & ${ }^{*} 9.78$ \\
\hline Column effects only & 9 & ${ }^{*} 11.37$ & 6 & ${ }^{*} 10.26$ & 6 & ${ }^{*} 12.29$ & 6 & 16.74 \\
\hline Row and column effects & 6 & ${ }^{*} 8.03$ & 4 & ${ }^{*} 1.29$ & 4 & ${ }^{*} 2.05$ & 4 & ${ }^{*} 9.31$ \\
\hline Row, column, interactions & 2 & ${ }^{*} 0.22$ & 1 & ${ }^{*} 0.31$ & 1 & ${ }^{*} 0.35$ & 1 & ${ }^{*} 0.94$ \\
\hline Saturated model & 0 & 0.00 & 0 & ${ }^{*} 0.00$ & 0 & 0.00 & 0 & 0.00 \\
\hline \multicolumn{9}{|l|}{ RC models } \\
\hline Independence & 12 & ${ }^{*} 14.20$ & 9 & 141.95 & 9 & 158.15 & 9 & 17.12 \\
\hline Constant association & 11 & ${ }^{*} 12.04$ & 8 & 19.46 & 8 & 48.66 & 8 & 16.71 \\
\hline Row effects only & 8 & ${ }^{*} 8.21$ & 6 & 12.90 & 6 & 18.84 & 6 & ${ }^{*} 11.69$ \\
\hline Column effects only & 9 & ${ }^{*} 11.88$ & 6 & 14.35 & 6 & 45.12 & 6 & 15.14 \\
\hline Row and column effects & 6 & ${ }^{*} 2.22$ & 4 & ${ }^{*} 5.16$ & 4 & 10.48 & 4 & ${ }^{*} 1.44$ \\
\hline Saturated model & 0 & 0.00 & 0 & 0.00 & 0 & 0.00 & 0 & 0.00 \\
\hline
\end{tabular}

Next, we turn to the psychiatric study. Table 7 summarizes the deviance $\chi^{2}$ goodness-of-fit statistics for the models fitted to Tables 2-5.

Table 2 shows a complete lack of association. As a consequence, the independence model is accepted for both the Dale and the RC model. Of course, the deviance for the independence model in both families is exactly the same. Initial severity measures symptoms present at baseline, whereas side effects measures symptoms induced by the therapy. Thus, the independence model implies that incidence and intensity of side effects do not depend on the initial conditions. Note that for Tables $2-5$ the $\mathrm{R}+\mathrm{C}+\mathrm{RC}$ model is overparameterized and thus coincides with the saturated model, whence it is not included in Table 7. 
Table 8

Psychiatric study: global cross ratios for the classification of side effects at Time 2 versus Time 3 (Data in Table 4)

\begin{tabular}{lccc}
\hline Side 2 & \multicolumn{3}{c}{ Side 3} \\
\cline { 2 - 4 } & 1 & 2 & 3 \\
\hline Observed & & & \\
1 & 21.15 & $+\infty$ & $+\infty$ \\
2 & 6.00 & 31.37 & 41.74 \\
3 & 1.17 & 6.05 & 43.17 \\
\multicolumn{4}{l}{ Row and column effects } \\
1 & 21.07 & 116.88 & \\
2 & 5.70 & 31.65 & 200.06 \\
3 & 1.20 & 6.67 & 43.26 \\
\hline
\end{tabular}

For Table 3 we find a strong association main effect with the Dale model. The constant global cross-ratio is very high: $\hat{\psi}=\hat{\psi}_{i j}=\exp (2.52)=12.43$. Note that this model corresponds to an underlying Plackett (1965) distribution, as such a distribution is characterized by a constant global cross-ratio. The fit improves by 7.68 on 2 degrees of freedom if we add a row effect. This model deserves our preference. For the RC family, there is certainly a strong constant association effect, but the fit is not acceptable at that point. A fully satisfactory fit is provided by the row and column association model.

There is also a clear global association main effect in Table 4. Including this parameter improves the fit of the model dramatically, although adding both row and column effects provides a better fit. Associations are shown in Table 8. Some of the observed cross-ratios are infinite, due to zero cells in the contingency table. All but one associations are high to extremely high. High associations in the upper right corner are explained by the fact that side effects over time are of course highly correlated, but also tend to go down, and only rarely go up, showing that the drug has a beneficial effect. It is remarkable that no RC model fits the data well, as can be learnt from Table 7. In conclusion, a marginal model such as the Dale model fits the data better than a model from the RC family. Should one choose to remain within the RC family, then a model of a more elaborate nature, such as the ones discussed in Section 5, might be needed. Note, again, that the $\mathrm{R}+\mathrm{C}+\mathrm{RC}$ model is no alternative, since it is overparameterized. Fitting related model (2.6) to Table 5 yields an acceptable fit: $\chi^{2}=6.33$ on 4 degrees of freedom $(P=0.1760)$.

Both Tables 3 and 4 are cross-classifications of an ordinal variable, recorded at two subsequent measurement times. In both cases, a parsimonious global association model explains the data well. It seems to be much harder to fit these data with local association models.

For Table 5, the row effects model is the most parsimonious one that provides an acceptable fit. One might argue that it is careful to retain the model adding column effects and interactions as well. Therefore, fitted frequencies for both models are shown in Table 5. Table 9 shows the global cross-ratios for the data of Table 5, 
Table 9

Psychiatric study: global cross ratios for the classification of side effects versus therepautic effect (both at the second occasion)

\begin{tabular}{llll}
\hline Side 2 & \multicolumn{3}{c}{ Therapeutic 2} \\
\cline { 2 - 4 } & 1 & 2 & 3 \\
\hline Observed & & & \\
1 & 0.97 & 0.95 & 0.74 \\
2 & 0.61 & 1.33 & 2.12 \\
3 & 0.41 & 2.57 & 4.26 \\
Column effects only & & \\
1 & 0.86 & 0.86 & 0.86 \\
2 & 1.77 & 1.77 & 1.77 \\
3 & 3.24 & 3.24 & 3.24 \\
Row, column, interaction & & \\
1 & 0.92 & 0.86 & 0.80 \\
2 & 0.55 & 1.55 & 1.92 \\
3 & 0.37 & 2.17 & 4.00 \\
\hline
\end{tabular}

together with the predicted values under both models. We observe two patterns in Table 9. First, the association increases along the main diagonal. This means that the association between the variables $I$ (SIDE2 $\leq 1$ ) and $I$ (THER $2 \leq 1$ ) is smaller than the association between the variables $I(\operatorname{SIDE} 2 \leq 3)$ and $I$ (THER $2 \leq 3)$. Also, the association becomes "negative" (i.e. smaller than 1 on the cross-ratio scale) for pairs such as $I$ (SIDE2 $\leq 3$ ) and $I$ (THER $2 \leq 1$ ). The RC models, fitted to this table, suggest the selection of the row and column effects model. The fitted model is also presented in Table 5 .

All RC models are based on model (2.4).

In conclusion, the Dale model yields a non-linear association model for Tables 1 and 5, through the interaction terms in (2.14), which is a very natural association model since it is a Dale model analogue of Goodman's R+C+RC model, of which the cross-ratios are given by (2.9). For Tables $2-4$, simpler association models, including at most row and/or column effects, but no interactions, are found to be acceptable. The models of RC type fitted to these data tend to be of a more complex nature, arguably since they model the association through local cross-ratios even though the data are ordered categorical.

\section{Extensions}

As mentioned earlier, the psychiatric study recorded more than two outcomes and further there is covariate information available. We consider in turn two ways of extending the models described sofar. First, we discuss the inclusion of covariates in marginal association models, followed by a generalization to multi-way tables. These extensions are members of the class (2.3). 
Table 10

Deviance $\chi^{2}$ goodness-of-fit statistics for Dale models, fitted to Table 5 (distinguishing between sex groups). Models with an acceptable fit are indicated by an asterisk

\begin{tabular}{llrr}
\hline Marginal model & Association model & df & \multicolumn{1}{c}{$\chi^{2}$} \\
\hline No sex effect & Constant & 23 & 39.32 \\
No sex effect & Sex, row & 20 & $* 27.76$ \\
No sex effect & Sex, row, column & 18 & $* 27.30$ \\
Sex effect & Constant & 21 & 36.80 \\
Sex effect & Sex effect & 20 & 31.74 \\
Sex effect & Sex, row, column & 16 & $* 25.53$ \\
Saturated & Saturated & 0 & 0.00 \\
\hline
\end{tabular}

\subsection{Covariates}

The marginal Dale model presented here is flexible in incorporating covariate effects. Their influence on the marginal means and on the association can be described in separate ways. For example, age could be found to influence the marginal response functions, while the association could be seen to change with sex. We will exemplify the possibilities that are brought about by this feature using two covariates. First, the data presented in Table 5 are split into two sex groups. Secondly, we will add the effect of the continuous covariate age on the responses and on the association between responses.

Let us consider sex first. Selected models, fitted to these data, are presented in Table 10. Obviously the marginal regressions are independent of sex, but we do find a sex effect in the association. If we add row effects (but no column effects), the fit is satisfactory $(P=0.12)$. The association structure of this model is

$$
\ln \psi_{i j r}=1.64-0.88 \operatorname{sex}_{r}-1.24 I(i=1)-0.56 I(i=2),
$$

where $\psi_{i j r}$ is the global cross-ratio, depending on subject $r$ through their sex, and $I($.$) is the indicator function. The association is stronger for males than for females$ $(P=0.0402)$.

Even though Table 5 (split by sex) contains four sampling zeros, no convergence problems are encountered and all parameters lie in the interior of their space. The Dale likelihood attains its maximum in the interior of the parameter space under very mild conditions, a feature shared with univariate ordinal logistic regression, which it generalizes. First, there must not be a complete separation in the covariate space between response groups. A similar condition was derived for the multigroup logistic model by Albert and Lesaffre (1986). Secondly, even with zero cell counts, models can be constructed for which the MLE lies in the interior of the space. For example, in a $3 \times 3$ table with cells $(1,1),(1,3),(2,2),(3,1)$, and $(3,3)$ equal to zero (with the other cells non-zero), a model with global cross-ratio dependent on row and column classification, yields finite estimates. Clearly, these properties simplify inclusion of continuous covariates, such as age. For 296 subjects out of 299 recorded in Table 5, age (in yr) is recorded. Age ranges from 16 to $75 \mathrm{yr}$, with a mean of $42.2 \mathrm{yr}$ (median is $40.5 \mathrm{yr}$ ). There are 97 distinct age by sex combinations, which yields an average of 
Table 11

Backward selection for Dale models, fitted to Table 5 (including sex and age). The number of model parameters (Par), the deviance (Dev) of the model are reported. For each model comparison, the reference model (Comp), and the corresponding $\chi^{2}$ statistic and $P$ value are reported

\begin{tabular}{llllllllll}
\hline $\mathrm{Nr}$ & Side 2 & Ther. 2 & Association & Par & Dev & Comp & df & $\chi^{2}$ & P \\
\hline 1 & Sex, age & Sex, age & Sex, age, row, col. & 17 & 1372.50 & & & & \\
2 & Age & - & Sex, age, row, col. & 14 & 1375.02 & 1 & 3 & 2.52 & 0.472 \\
3 & Age & - & Sex, age, row & 12 & 1375.52 & 2 & 2 & 0.50 & 0.779 \\
4 & Age & - & Age, row & 11 & 1378.99 & 3 & 1 & 3.47 & 0.063 \\
5 & Age & - & Sex, row & 11 & 1379.01 & 3 & 1 & 3.49 & 0.062 \\
6 & Age & - & Row & 10 & 1382.65 & 4 & 1 & 3.66 & 0.056 \\
6 & Age & - & Row & 10 & 1382.65 & 3 & 2 & 7.13 & 0.028 \\
\hline
\end{tabular}

about 3 subjects per distinct $4 \times 4$ table! Thus, we have a generalization of a purely contingency table analysis to multivariate ordinal regression. Obviously, a saturated model is not meaningful here, since the number of covariate levels (and hence the number of cells) increases with the sample size. Derivation of formal goodness-offit tools, such as appropriate residuals, requires further research. The most complex model we will consider, contains sex and age effects in both the marginal mean and in the association and lets the association further depend on row and column classification. Clearly, this model could be extended (for example, by means of higher-order effects of age and interactions between sex and age). Table 11 reports on a backward selection performed to simplify the model. In the final model, the marginal logits are simplified such that only SIDE2 depends on age. The association is independent of the column classification. Although sex and age could be omitted from the association when comparing models 4 and 5 with 3 , or 6 with 4 and 5 , a direct comparison of model 6 (no covariate influence on association) with model 3 (both age and sex influence the association) is significant at the $5 \%$ level. Therefore, we prefer model 3. The cumulative logits (2.10) and (2.11) for subject $r$ are

$$
\begin{aligned}
& \eta_{1 i r}=0.54 I(i=1)+2.63 I(i=2)+3.75 I(i=3)-0.019 \text { age }_{r}, \\
& \eta_{2 j r}=-2.69 I(j=1)-0.47 I(j=2)+0.95 I(j=3),
\end{aligned}
$$

and the association structure is

$$
\ln \psi_{i j r}=2.94-0.80 \mathrm{sex}_{r}-0.028 \mathrm{age}_{r}-1.44 I(i=1)-0.63 I(i=2) .
$$

The logit for side effects decreases with age, implying e.g., that the probability of category 1 (no side effects) decreases and the probability of category 4 (highest level of side effects) increases with age. The association is stronger for males than for females (consistent with Table 10) and decreases with age.

\subsection{Multi-way contingency tables}

Molenberghs and Lesaffre (1994) extended the Dale model, originally constructed for two response variables, to arbitrary dimensions. This implies that the model is 
suitable to analyze multi-way contingency tables. Computational details can be found in Molenberghs and Lesaffre (1994). We illustrate this technique on the psychiatric data set, for a cross-classification of therapeutic effect at visits 2,3 , and 4 . The data are presented as a $4 \times 4 \times 4$ contingency table (Table 12). There are 242 patients with measurements on all three outcomes. Let the variables $Y_{1}, Y_{2}$, and $Y_{3}$ have $I, J$, and $K$ levels, respectively, and define cumulative three-way probabilities $\mu_{i j k}$ $(i=1, \ldots, I ; j=1, \ldots, J ; k=1, \ldots, K)$, similar to the definition in Section 2 . The

Table 12

Psychiatric study: cross-classification of therapeutic effect at the second, third, and fourth occasion. The fitted values are in parentheses. The first entry corresponds to Model 1, the second entry corresponds to Model 2, the third entry corresponds to the generalized RC model

\begin{tabular}{|c|c|c|c|c|c|}
\hline \multirow[b]{2}{*}{ Side 2} & \multirow[b]{2}{*}{ Side 3} & \multicolumn{4}{|c|}{ Side 4} \\
\hline & & 1 & 2 & 3 & 4 \\
\hline \multirow[t]{4}{*}{1} & 1 & $\begin{array}{l}11 \\
(10.18) \\
(13.75) \\
(10.99)\end{array}$ & $\begin{array}{c}1 \\
(1.19) \\
(2.07) \\
(0.48)\end{array}$ & $\begin{array}{c}0 \\
(0.10) \\
(0.18) \\
(0.00)\end{array}$ & $\begin{array}{c}0 \\
(0.02) \\
(0.05) \\
(0.00)\end{array}$ \\
\hline & 2 & $\begin{array}{c}0 \\
(0.60) \\
(0.89) \\
(1.16)\end{array}$ & $\begin{array}{c}1 \\
(1.46) \\
(1.88) \\
(0.97)\end{array}$ & $\begin{array}{c}1 \\
(0.30) \\
(0.40) \\
(0.02)\end{array}$ & $\begin{array}{c}0 \\
(0.06) \\
(0.10) \\
(0.00)\end{array}$ \\
\hline & 3 & $\begin{array}{l}0 \\
(0.05) \\
(0.08) \\
(0.05)\end{array}$ & $\begin{array}{l}0 \\
(0.18) \\
(0.21) \\
(0.15)\end{array}$ & $\begin{array}{l}0 \\
(0.15) \\
(0.13) \\
(0.07)\end{array}$ & $\begin{array}{l}0 \\
(0.06) \\
(0.05) \\
(0.01)\end{array}$ \\
\hline & 4 & $\begin{array}{l}0 \\
(0.01) \\
(0.02) \\
(0.01)\end{array}$ & $\begin{array}{c}0 \\
(0.03) \\
(0.05) \\
(0.02)\end{array}$ & $\begin{array}{l}0 \\
(0.04) \\
(0.04) \\
(0.03)\end{array}$ & $\begin{array}{l}0 \\
(0.02) \\
(0.02) \\
(0.03)\end{array}$ \\
\hline \multirow[t]{4}{*}{2} & 1 & $\begin{array}{l}33 \\
(36.27) \\
(30.39) \\
(32.34)\end{array}$ & $\begin{array}{l}2 \\
(2.92) \\
(3.18) \\
(2.76)\end{array}$ & $\begin{array}{c}0 \\
(0.33) \\
(0.35) \\
(0.00)\end{array}$ & $\begin{array}{c}0 \\
(0.08) \\
(0.09) \\
(0.00)\end{array}$ \\
\hline & 2 & $\begin{array}{l}13 \\
(13.80) \\
(13.03) \\
(16.88)\end{array}$ & $\begin{array}{l}23 \\
(18.28) \\
(16.81) \\
(17.72)\end{array}$ & $\begin{array}{l}2 \\
(2.14) \\
(2.19) \\
(0.73)\end{array}$ & $\begin{array}{l}0 \\
(0.39) \\
(0.46) \\
(0.00)\end{array}$ \\
\hline & 3 & $\begin{array}{l}1 \\
(0.47) \\
(0.44) \\
(1.48)\end{array}$ & $\begin{array}{c}2 \\
(1.84) \\
(1.31) \\
(4.48)\end{array}$ & $\begin{array}{l}3 \\
(1.87) \\
(1.05) \\
(2.39)\end{array}$ & $\begin{array}{l}0 \\
(0.62) \\
(0.40) \\
(0.24)\end{array}$ \\
\hline & 4 & $\begin{array}{c}0 \\
(0.10) \\
(0.10) \\
(0.20)\end{array}$ & $\begin{array}{l}1 \\
(0.30) \\
(0.29) \\
(0.83)\end{array}$ & $\begin{array}{l}1 \\
(0.34) \\
(0.22) \\
(0.99)\end{array}$ & $\begin{array}{c}1 \\
(0.21) \\
(0.11) \\
(0.95)\end{array}$ \\
\hline
\end{tabular}


Table 12 (contd.)

\begin{tabular}{|c|c|c|c|c|c|}
\hline \multirow[b]{2}{*}{ Side 2} & \multirow[b]{2}{*}{ Side 3} & \multicolumn{4}{|c|}{ Side 4} \\
\hline & & 1 & 2 & 3 & 4 \\
\hline \multirow[t]{4}{*}{3} & 1 & $\begin{array}{l}12 \\
(8.39) \\
(7.41) \\
(12.59)\end{array}$ & $\begin{array}{l}1 \\
(1.04) \\
(1.12) \\
(1.70)\end{array}$ & $\begin{array}{l}0 \\
(0.16) \\
(0.17) \\
(0.00)\end{array}$ & $\begin{array}{l}0 \\
(0.04) \\
(0.05) \\
(0.00)\end{array}$ \\
\hline & 2 & $\begin{array}{l}25 \\
(22.86) \\
(24.97) \\
(19.74)\end{array}$ & $\begin{array}{l}25 \\
(24.33) \\
(28.79) \\
(24.31)\end{array}$ & $\begin{array}{l}1 \\
(1.45) \\
(2.09) \\
(1.48)\end{array}$ & $\begin{array}{l}1 \\
(0.27) \\
(0.38) \\
(0.00)\end{array}$ \\
\hline & 3 & $\begin{array}{l}1 \\
(1.15) \\
(1.33) \\
(2.75)\end{array}$ & $\begin{array}{c}8 \\
(10.76) \\
(9.96) \\
(8.61)\end{array}$ & $\begin{array}{l}5 \\
(7.30) \\
(6.61) \\
(4.98)\end{array}$ & $\begin{array}{l}1 \\
(1.56) \\
(1.64) \\
(0.63)\end{array}$ \\
\hline & 4 & $\begin{array}{l}0 \\
(0.22) \\
(0.30) \\
(0.43)\end{array}$ & $\begin{array}{l}3 \\
(0.83) \\
(1.05) \\
(1.78)\end{array}$ & $\begin{array}{l}0 \\
(1.16) \\
(1.02) \\
(2.07)\end{array}$ & $\begin{array}{l}0 \\
(0.97) \\
(0.71) \\
(1.91)\end{array}$ \\
\hline \multirow[t]{4}{*}{4} & 1 & $\begin{array}{c}1 \\
(1.96) \\
(1.42) \\
(0.08)\end{array}$ & $\begin{array}{l}0 \\
(0.42) \\
(0.36) \\
(0.05)\end{array}$ & $\begin{array}{l}0 \\
(0.07) \\
(0.06) \\
(0.00)\end{array}$ & $\begin{array}{l}0 \\
(0.02) \\
(0.02) \\
(0.00)\end{array}$ \\
\hline & 2 & $\begin{array}{l}5 \\
(8.87) \\
(8.16) \\
(5.50)\end{array}$ & $\begin{array}{c}6 \\
(5.58) \\
(5.55) \\
(11.75)\end{array}$ & $\begin{array}{l}0 \\
(0.46) \\
(0.44) \\
(2.70)\end{array}$ & $\begin{array}{l}0 \\
(0.11) \\
(0.11) \\
(0.03)\end{array}$ \\
\hline & 3 & $\begin{array}{l}7 \\
(3.07) \\
(3.26) \\
(3.82)\end{array}$ & $\begin{array}{l}18 \\
(19.88) \\
(19.69) \\
(13.39)\end{array}$ & $\begin{array}{c}9 \\
(7.74) \\
(6.67) \\
(10.15)\end{array}$ & $\begin{array}{l}1 \\
(0.99) \\
(0.97) \\
(2.78)\end{array}$ \\
\hline & 4 & $\begin{array}{l}0 \\
(0.51) \\
(0.68) \\
(0.98)\end{array}$ & $\begin{array}{l}2 \\
(3.13) \\
(4.42) \\
(3.98)\end{array}$ & $\begin{array}{l}8 \\
(7.48) \\
(7.84) \\
(4.37)\end{array}$ & $\begin{array}{l}6 \\
(4.78) \\
(4.45) \\
(3.41)\end{array}$ \\
\hline
\end{tabular}

model extends as follows. Apart from three sets of marginal parameters, one for each measurement time:

$$
\begin{aligned}
& \eta_{1 i}=\operatorname{logit}\left(\operatorname{pr}\left(Y_{1} \leq i\right)\right)=\ln \left(\mu_{i J K}\right)-\ln \left(1-\mu_{i J K}\right), \\
& \eta_{2 j}=\operatorname{logit}\left(\operatorname{pr}\left(Y_{2} \leq j\right)\right)=\ln \left(\mu_{I j K}\right)-\ln \left(1-\mu_{I j K}\right), \\
& \eta_{3 k}=\operatorname{logit}\left(\operatorname{pr}\left(Y_{3} \leq k\right)\right)=\ln \left(\mu_{I J k}\right)-\ln \left(1-\mu_{I J k}\right)
\end{aligned}
$$


$(i=1, \ldots, I-1 ; j=1, \ldots, J-1 ; k=1, \ldots, K-1)$, there are also three sets of pairwise association parameters:

$$
\begin{aligned}
& \ln \psi_{12, i j}=\ln \frac{\mu_{i j K}\left(1-\mu_{I j K}-\mu_{i J K}+\mu_{i j K}\right)}{\left(\mu_{i J K}-\mu_{i j K}\right)\left(\mu_{I j K}-\mu_{i j K}\right)}, \\
& \ln \psi_{13, i k}=\ln \frac{\mu_{i J k}\left(1-\mu_{I J k}-\mu_{i J K}+\mu_{i J k}\right)}{\left(\mu_{i J K}-\mu_{i J k}\right)\left(\mu_{I J k}-\mu_{i J k}\right)}, \\
& \ln \psi_{23, j k}=\ln \frac{\mu_{I j k}\left(1-\mu_{I J k}-\mu_{I j K}+\mu_{I j k}\right)}{\left(\mu_{I J k}-\mu_{I j k}\right)\left(\mu_{I j K}-\mu_{I j k}\right)}
\end{aligned}
$$

together with a set of three-way associations (generalized cross-ratios):

$\ln \psi_{123, i j k}=$

$\ln \frac{\mu_{i j k}\left(\mu_{i J K}-\mu_{i j K}-\mu_{i J k}+\mu_{i j k}\right)\left(\mu_{I j K}-\mu_{i j K}-\mu_{I j k}+\mu_{i j k}\right)\left(\mu_{I J k}-\mu_{i J k}-\mu_{I j k}+\mu_{i j k}\right)}{\left(\mu_{i j K}-\mu_{i j k}\right)\left(\mu_{i J k}-\mu_{i j k}\right)\left(\mu_{I j k}-\mu_{i j k}\right)\left(1-\mu_{i J K}-\mu_{I j K}-\mu_{I J k}+\mu_{i j K}+\mu_{i J k}+\mu_{I j k}-\mu_{i j k}\right)}$.

Clearly, the link functions (4.16)-(4.22) are all expressed in terms of constrasts of log-probabilities, and hence fit in (2.1). Molenberghs and Lesaffre (1994, 1997b) describe ways to determine the joint probabilities $\mu_{i j k}$ from the links and to compute maximum likelihood estimates. Indeed, the key issue in a marginal model of this type is the construction of the joint probabilities. The univariate marginal probabilities $\mu_{i J K}, \mu_{I j K}$, and $\mu_{I J k}$ are easily determined from inverting (4.16)-(4.18), just as with (2.10) and (2.11). The pairwise marginal probabilities $\mu_{i j K}, \mu_{i J k}$, and $\mu_{i j k}$, can be written in analogy with (2.15), since links (4.19)-(4.21) have the same form as (2.12). Determining the third-order cumulative probabilities $\mu_{i j k}$ is more difficult. Several proposals have been made. Molenberghs and Lesaffre (1994) solve defining polynomials to obtain the probabilities as well as their first and second order derivatives with respect to the parameters of interest to construct the multinomial log-likelihood, the score equations, and the Hessian matrix, respectively. Lang and Agresti (1994) solved so-called constrained equations and Glonek and McCullagh (1995) used a Newton iteration scheme. Both authors use a version of the NewtonRaphson iteration procedure to maximize the likelihood. Finally, Molenberghs and Lesaffre (1997b) adapted the iterative proportional fitting algorithm (Deming and Stephan, 1940), typically used to fit log-linear models (which are defined in terms of conditional probabilities) to the current marginal setting. Fitzmaurice and Laird (1993) used the iterative proportional fitting algorithm to determine the probabilities in their mixed marginal-conditional model.

To illustrate the model, let us analyze the three therapeutic effect measurements. Model 1 assumes the marginal logits (4.16)-(4.18) are independent of covariate effects, yielding nine marginal parameters. Each of the association parameters $\psi$ in (4.19)-(4.22) is assumed independent of covariate effects as well as of the category indicators $i, j$, and $k$, yielding three pairwise and one three-way association parameters. This brings the total number of parameters to 13. Marginal parameter 
estimates (standard errors in parentheses) are

$$
\begin{array}{lll}
\hat{\eta}_{11}=-2.76(0.27), & \hat{\eta}_{21}=-1.04(0.14), & \hat{\eta}_{31}=-0.21(0.13), \\
\hat{\eta}_{12}=-0.45(0.13), & \hat{\eta}_{22}=0.75(0.13), & \hat{\eta}_{32}=1.58(0.17), \\
\hat{\eta}_{13}=1.00(0.15), & \hat{\eta}_{23}=2.40(0.22), & \hat{\eta}_{33}=3.12(0.32) .
\end{array}
$$

The constant global cross-ratios are $\hat{\psi}_{12}=\hat{\psi}_{12, i j}=\exp (2.58)=13.18(3.08)$ for the first and the second outcome, $\hat{\psi}_{13}=\hat{\psi}_{13, i k}=\exp (1.38)=3.99(0.89)$ for the first and the third outcome, and $\hat{\psi}_{23}=\hat{\psi}_{23, j k}=\exp (3.08)=21.76(5.74)$ for the second and the third outcome. The three-way interaction, $\hat{\psi}_{123}=\hat{\psi}_{123, i j k}=\exp (0.18)=1.19(0.66)$, is not significantly different from 1 . Fitted frequencies are given in Table 12.

The overall deviance goodness-of-fit statistic is 37.13 on 50 degrees of freedom $(P=0.9115)$. Inspecting standardized residuals, 62 out of 64 are less than 2 in absolute value, the remaining ones being 2.24 and 2.39 . Thus, model fit is acceptable, but one might want to simplify the model further. We will in turn simplify the marginal and association structures. First, the three sets of logits reveal an increase over time, suggesting an improving response to therapy. A simpler model would assume: $\eta_{1 i}=\alpha_{i}, \eta_{2 j}=\alpha_{j}+\pi_{2}$, and $\eta_{3 k}=\alpha_{k}+\pi_{3}(i, j, k=1,2,3)$. We interpret $\alpha_{1}, \alpha_{2}$, and $\alpha_{3}$ as cut-off points at the first occasion and $\pi_{2}$ and $\pi_{3}$ as "proportional" shift parameters at occasions 2 and 3 respectively. Secondly, one might argue that the association between outcomes is mainly a function of the time lag between the outcomes, but not so much of the measurement times themselves. This is supported by the fact that $\ln \psi_{12}$ and $\ln \psi_{23}$ are roughly the same (given their standard errors of about 0.24 ), with $\ln \psi_{13}$ approximately half of the other association. Should one grant belief to this assumption, then an association model of the form $\gamma=\ln \psi_{12}=0.5 \ln \psi_{13}=\ln \psi_{23}$ might be considered. The multiplier 0.5 for $\ln \psi_{13}$ is suggested by the data and has limited empirical or theoretical support. Alternatively, one could estimate this parameter from the data. Thirdly, the three-way interaction can be set to zero. There are six parameters in total.

Parameter estimates (standard errors) for this model are $\hat{\alpha}_{1}=-2.41(0.17)$, $\hat{\alpha}_{2}=-0.52(0.12), \hat{\alpha}_{3}=1.02(0.14)$ for the cut-off points, with time shifts $\hat{\pi}_{2}=$ $1.32(0.11)$ for the second period and $\hat{\pi}_{3}=2.17(0.16)$ for the third period. The single association parameter is equal to $\hat{\gamma}=2.81(0.17)$, resulting in $\hat{\psi}_{12}=\psi_{23}=16.56(2.77)$ and $\hat{\psi}_{13}=\sqrt{16.56}=4.07(0.34)$. Fitted frequencies are given in Table 12. This model has a deviance of 43.67 on 57 degrees of freedom $(P=0.9029)$, and again only two standardized residuals are larger than 2 (being 2.07 and 2.70), showing that there is some support in the data for the assumed model. Finally, comparing Models 1 and 2 yields a deviance of 6.54 on 7 degrees of freedom $(P=0.4782)$, indicating that the first and more complex model is not necessary.

A similar model is obtained from the analysis of side effects at times 2,3 , and 4. Analyzing initial severity, side effects at time 2, and therapeutic effect at time 2 , yields a satisfactory model with only constant association. No details on these models are included. These results are promising since they support the thesis that 
for a range of ordinal data applications, parsimonious marginal global cross-ratio models are sufficient to describe the data.

In case nominal data are to be analyzed, then the model can be adapted to cell probabilities $\mu_{i j k}^{*}$. This would mean that Eqs. (4.16)-(4.22) have to be changed in the spirit of (2.13). In particular, the global cross-ratios might have to be replaced by their local counterparts.

In addition to the extensions studied so far, it is possible to extend the RC model to more than two dimensions. One option is to extend model (2.4) by defining

$$
\mu_{i j k}^{*}=\alpha_{i} \beta_{j} \gamma_{k} \mathrm{e}^{\phi \lambda_{i} v_{j} \omega_{k}}
$$

with obvious notation. Of course, the marginal pairwise local odds ratio for a pair $(i, j)$ has a very complicated form and $(2.4)$ is not a submodel of (4.23) in the sense that the interpretation of the parameters will change in passing from a bivariate to a trivariate model. The conditional pairwise odds ratio on the other hand is $\ln \theta_{i j \mid k}^{*}=\phi \omega_{k}\left(\lambda_{i}-\lambda_{i+1}\right)\left(v_{j}-v_{j+1}\right)$, where $\omega_{k}$ can be considered as an adjustment for the category conditioned upon. The three-way odds ratio is similar in structure to the two-way odds ratio of the bivariate model (2.4).

Fitting model (4.23) to the trivariate therapeutic data of Table 12 yields a deviance of 67.96 on 47 degrees of freedom $(P=0.0243)$, indicating that the fit is not satisfactory. Fitted frequencies are displayed in Table 12 . One could consider more elaborate alternatives, such as trivariate versions of the $\mathrm{R}+\mathrm{C}+\mathrm{RC}$ model (2.8). However, as indicated earlier, for this kind of data, the marginal model defined in terms of cumulative probabilities seems to be more promising, since it yields very parsimonious descriptions of the association structure.

An alternative fashion to extend (2.4) would start from three pairwise marginal RC models:

$$
\begin{aligned}
& \mu_{i j+}^{*}=\alpha_{i}^{(12)} \beta_{j}^{(12)} \mathrm{e}^{\phi^{(12)} \lambda_{i}^{(12)} v_{j}^{(12)}}, \\
& \mu_{i+k}^{*}=\alpha_{i}^{(13)} \gamma_{k}^{(13)} \mathrm{e}^{\phi^{(13)} \lambda_{i}^{(13)} \tau_{k}^{(13)}}, \\
& \mu_{+j k}^{*}=\beta_{j}^{(23)} \gamma_{k}^{(23)} \mathrm{e}^{\phi^{(23)} v_{j}^{(23)} \tau_{k}^{(23)}}
\end{aligned}
$$

$(i=1, \ldots, I ; j=1, \ldots, J ; k=1, \ldots, K)$. In order for $(4.24)-(4.26)$ to define a valid probability mass function $\mu_{i j k}^{*}$, complicated restrictions must be satisfied. Indeed, summing (4.24) over $j$ and (4.25) over $k$ yields $I$ restrictions:

$$
\alpha_{i}^{(12)} \sum_{j=1}^{J} \beta_{j}^{(12)} \mathrm{e}^{\phi^{(12)} \lambda_{i}^{(12)} v_{j}^{(12)}}=\alpha_{i}^{(13)} \sum_{k=1}^{K} \gamma_{k}^{(13)} \mathrm{e}^{\phi^{(13)} \lambda_{i}^{(13)} \tau_{k}^{(13)}}
$$

( $i=1, \ldots, I$ ), with similarly $J$ and $K$ restrictions for the other two marginals. The reason is that the parameters in the RC models are not marginal and hence are not reproducible in the sense of Liang et al. (1992). 


\section{Relation to latent continuous densities}

Several publications are devoted to the comparison of local and global association models. Important references are Goodman (1981b), Mardia (1970), Dale (1984) and Becker (1989). An argument, often used to claim superiority of local over global association models, is the close relationship between Goodman's UM model and discretizations of the bivariate normal distribution (Goodman, 1981b; Becker, 1989). Also, their close connection with log-linear modelling is brought forward as an argument in favour.

Holland and Wang (1987) introduced the local dependence function (LDF) of a bivariate continuous density function $f$ as an analog to the local cross-ratios for contingency tables (Yule and Kendall, 1950). The probability of a rectangular cell around $(x, y)$ with edges $\mathrm{d} x$ and $\mathrm{d} y$ is approximated by $f(x, y) \mathrm{d} x \mathrm{~d} y$. For cells around $(x, y),(x, v),(u, y)$ and $(u, v)$, the log local cross-ratio is given by

$$
\theta(x, y ; u, v)=\ln \left(\frac{f(x, y) f(u, v)}{f(x, v) f(u, y)}\right) .
$$

The local dependence function (LDF) at $(x, y)$ is defined as

$$
\gamma_{f}(x, y)=\lim _{\mathrm{d} x \rightarrow 0, \mathrm{~d} y \rightarrow 0} \frac{\theta(x, y ; x+\mathrm{d} x, y+\mathrm{d} y)}{\mathrm{d} x \mathrm{~d} y}=\frac{\partial^{2}}{\partial x \partial y} \ln f(x, y) .
$$

Holland and Wang (1987) show that a bivariate density is characterized by its LDF and its two marginal densities. Further, a bivariate normal is characterized by a constant LDF and two normal marginal densities. Precise statements and proofs are found in Holland and Wang (1987).

The LDF of a normal density with correlation $\rho$ is equal to $\phi=\rho /\left(1-\rho^{2}\right)$. Exactly this quantity, together with appropriately chosen scores $\alpha_{i}, \beta_{j}, \lambda_{i}$ and $v_{j}$, are used by Becker (1989) to approximate the discretized normal by model (2.4). Note that a special version of the RC model, i.e. the UM model, implies a constant local cross-ratio. It can be observed from Wang (1987), who provides an alternative way of computing normal probabilities, that the local association model introduced by (2.4) and the bivariate normal go naturally together. This explains why the local association models fit far better the discretized normal than do global cross-ratio models. In general, local association models correspond to bivariate densities via the LDF.

An analogous relationship holds between the Dale model and the Plackett distribution (Plackett, 1965; Mardia, 1970). Indeed, if the global cross-ratio is constant (or in particular zero) throughout a contingency table, then it corresponds to a bivariate Plackett distribution (constant "Yulean association"). This was the case for the global association models, selected in the case of Tables 2 and 3.

However, we observed that model construction is restricted to neither a constant local association, nor a constant global association. Within the family (2.3), one can even consider non-linear association models. In particular, we considered various types of row and column effects, together with interactions. This suggests that the normal distribution and the Plackett distribution are not the only ones of interest as 

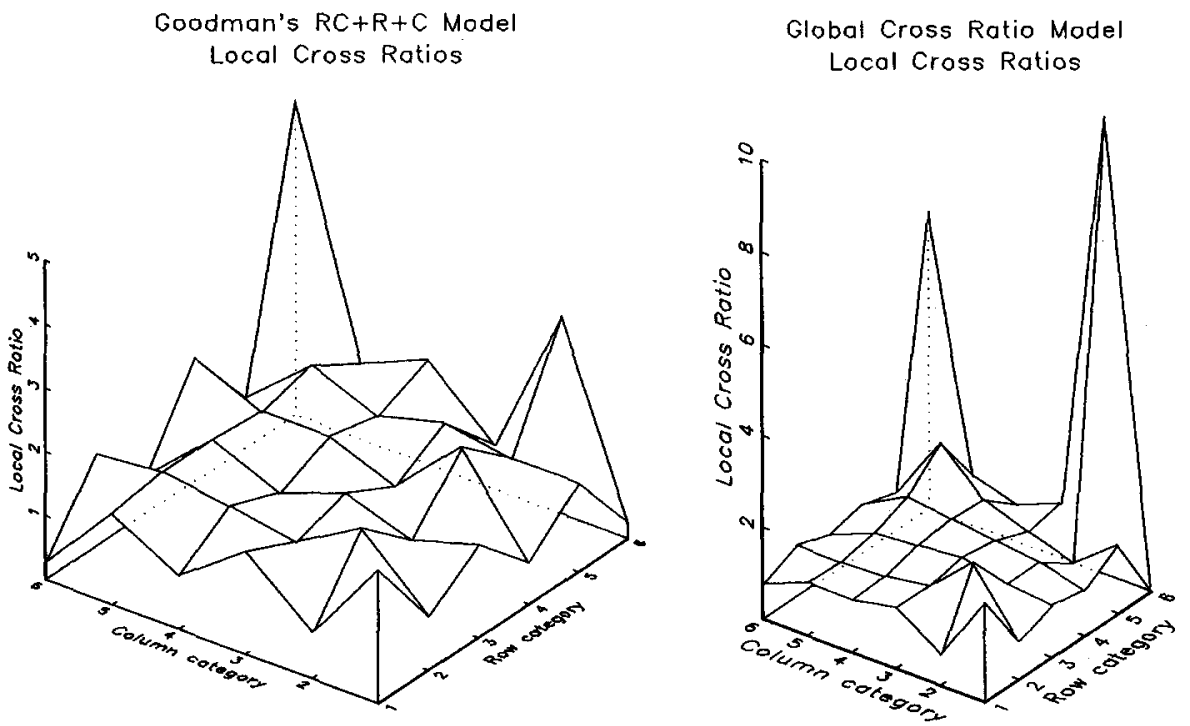

Goodman's $R C+R+C$ Model Global Cross Ratios

Global Cross Ratio Model Global Cross Ratios
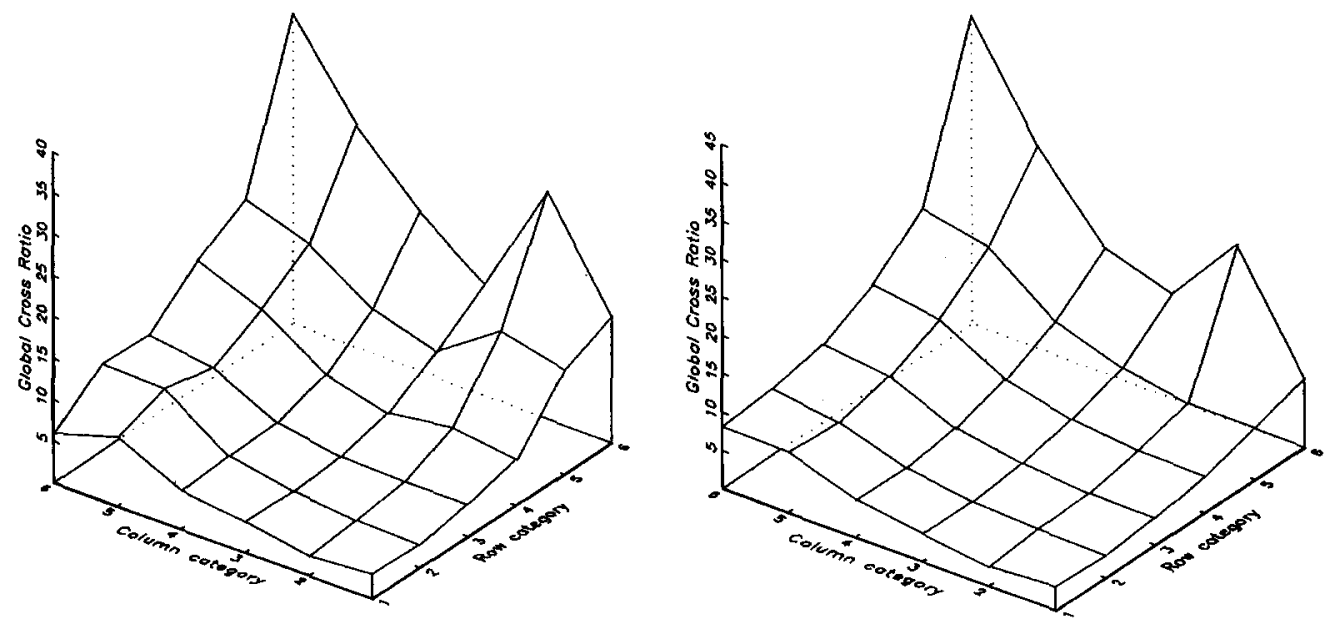

Fig. 1.

continuous distributions, underlying a contingency table. Different forms for the local and global cross-ratios correspond to different distributions.

The correspondence between contingency tables and distribution functions in the Dale model case is very easy. The definition of the distribution is found by the continuous version of (2.15), of which the explicit form is straightforward. A continuous version of (2.14) would include linear (and quadratic) terms in $x$ and $y$, together with an interaction term.

Let us turn attention again to Goodman's $\mathrm{R}+\mathrm{C}+\mathrm{RC}$ model (2.8). To construct a continuous density having a similar association structure, we first select a local 
dependence function of the form

$$
\gamma(\phi ; x, y)=\phi_{1}+\phi_{2} f_{2}(x)+\phi_{3} g_{3}(y)+\phi_{4} f_{4}(x) g_{4}(y),
$$

where $f_{k}$ and $g_{k}$ are integrable functions. Molenberghs and Lesaffre (1997a) show how the corresponding density can be approximated. The RC model is found by setting all terms, except those with subscript 4 , equal to zero.

The models, fitted to Table 1 , can be seen as extensions of both an underlying normal and an underlying Plackett distribution. The choice between different models should not be made on the ground of potential classes of underlying densities, but on the shape (structure) of associations. Fig. 1 presents local and global cross-ratios found from the fitted values of both the $\mathrm{RC}+\mathrm{R}+\mathrm{C}$ model and the global cross-ratio model with row and column effects, as well as interactions. Obviously, there is little pattern in the local cross-ratios, whereas the global cross-ratios show a clear tendency: all associations are high, with an increase if the dichotomy is constructed closer to the categories with low labels, being highest between the variables $I$ (Father's status $\leq 1$ ) and $I$ (Child's status $\leq 1$ ). This implies that social mobility increases with increasing category. There is also slight evidence that the association surface is symmetric, which would then correspond to a global cross-ratio distribution with symmetric global cross-ratio function, such as a symmetric second degree polynomial.

\section{Concluding remarks}

In this paper we presented association models for cross-classified data that belong to the unified multivariate logistic framework, described by McCullagh and Nelder (1989) and Glonek and McCullagh (1995). This family provides a versatile way of exploring the association structure of cross-classified data. It encompasses both local and global measures of association, with emphasis on cross-ratios, since log crossratios can be written as contrasts of log-probabilities. Both fully marginal models, such as the Dale model and its multivariate extensions, as models with a conditional flavour, such as Goodman's (1981a) RC model, are members of this family. Further, linear as well as non-linear link functions (e.g., involving interactions between row and column effects) link functions fit within this family.

We argue that, in spite of the close connection between an $\mathrm{RC}$ model and an underlying normal density and the absence of this connection with a fully marginal model, this last category of models provides a versatile way of exploring the association structure of cross-classified data, whether of nominal or of ordinal type. We infer from the examples that they often yield parsimonious descriptions of the association structure. Further, marginal association models are easily extended to marginal regression models to include covariate effects. Extensions to multi-way tables are possible, both with the $\mathrm{RC}$ as well as with the marginal family.

Both Dale (1984) and Anscombe (1981) suggest the use of global cross-ratios as soon as the outcomes are recorded as ordinal variables. We have shown in Sections 3 and 4.2 that this choice is supported by a very good fit for this kind of models to a range of applications. Further, we claim that the global cross-ratio can lead to 
interesting interpretations of the association structure itself, which we think is an often neglected aspect of data analysis.

An argument, in favour of RC models, is their computational simplicity. However, with the current state of high-quality statistical software, fitting marginal global association models poses no problems. In particular, we encountered no convergence problems. The calculations, necessary to carry out the fitting procedures for both types of models were performed with the statistical software packages GAUSS (Aptech, Inc.) and SPLUS (StatSci Division, MathSoft, Inc.).

\section{Acknowledgements}

We wish to thank the British Council and the Fonds voor Wetenschappelijk Onderzoek-Vlaanderen for their support. We thank also Solvay Duphar N.V. for the kind permission to use their data. Finally, we would like to thank the associate editor and anonymous referees for very constructive and helpful suggestions.

\section{References}

Albert, A., Lesaffre, E., 1986. Multiple group logistic discrimination. Comput. Math. Appl. 12, $209-224$. Anscombe, F.J., 1981. Computing in Statistical Science through APL. Springer, New York.

Bahadur, R.R., 1961. A representation of the joint distribution of responses to $n$ dichotomous items. In: Solomon, H. (Ed.), Studies in Item Analysis and Prediction. Stanford Mathematical Studies in the Social Sciences VI. Stanford University Press, Stanford, California, CA.

Becker, M.P., 1989. On the bivariate normal distribution and association models for ordinal categorical data. Statist. Probab. Lett. 8, 435-440.

Dale, J.R., 1984. Local versus global association for bivariate ordered responses. Biometrika 71, 507-514.

Dale, J.R., 1986. Global cross-ratio models for bivariate, discrete, ordered responses. Biometrics 42, 909-917.

Deming, W.E., Stephan, F.F., 1940. On a least squares adjustment of a sampled frequency table when the expected marginal totals are known. Ann. Math. Statist. 11, 427-444.

Fitzmaurice, G.M., Laird, N.M., 1993. A likelihood-based method for analysing longitudinal binary responses. Biometrika 80, 141-151.

Glonek, G.F.V., McCullagh, P., 1995. Multivariate logistic models. J. Roy. Statist. Soc. Ser. B 57, 533-546.

Goodman, L.A., 1969. How to ransack social mobility tables and other kinds of cross-classification tables. Amer. J. Sociol. 75, 1-39.

Goodman, L.A., 1979. Simple models for the analysis of association in cross-classifications having ordered categories. J. Amer. Statist. Assoc. 74, 537-552.

Goodman, L.A., 1981a. Association models and canonical correlation in the analysis of crossclassifications having ordered categories. J. Amer. Statist. Assoc. 76, 320-334.

Goodman, L.A., 1981b. Association models and the bivariate normal for contingency tables with ordered categories. Biometrika 68, 347-355.

Goodman, L.A., 1985. The 1983 Henry L. Rietz memorial lecture. The analysis of cross-classified data having ordered and/or unordered categories: association models, correlation models, and asymmetry models for contingency tables with or without missing entries. Ann. Statist. 13, 10-69.

Holland, P.W., Wang, Y.J., 1987. Dependence function for continuous bivariate densities. Comm. Statist. Theory Methods 2, 863-876. 
Lang, J.B., Agresti, A., 1994. Simultaneously modeling joint and marginal distributions of multivariate categorical responses. J. Amer. Statist. Assoc. 89, 625-632.

Lesaffre, E., Verbeke, G., Molenberghs, G., 1994. A sensitivity analysis of two multivariate response models. Comput. Statist. Data Anal. 17, 363-391.

Liang, K.-Y., Zeger, S.L., 1986. Longitudinal data analysis using generalized linear models. Biometrika $73,13-22$.

Liang, K.-Y., Zeger, S.L., Qaqish, B., 1992. Multivariate regression analyses for categorical data. J. Roy. Statist. Soc. Ser. B 54, 3-40.

Mardia, K.V., 1970. Families of Bivariate Distributions. Griffin, London.

McCullagh, P., Nelder, J.A., 1989. Generalized Linear Models. Chapman and Hall, London.

Molenberghs, G., Lesaffre, E., 1994. Marginal modelling of correlated ordinal data using a multivariate Plackett distribution. J. Amer. Statist. Assoc. 89, 633-644.

Molenberghs, G., Lesaffre, E., 1997a. Non-linear integral equations to construct bivariate densities with given marginals and dependence function. Statistica Sinica 7, 713-738.

Molenberghs, G., Lesaffre, E., 1997b. Marginal modelling of multivariate categorical data. Submitted for publication.

Plackett, R.L., 1965. A class of bivariate distributions. J. Amer. Statist. Assoc. 60, 516-522.

Wang, Y.J., 1987. The probability integrals of bivariate normal distributions: a contingency table approach. Biometrika 74, 185-190.

Yule, G.U., Kendall, M.G., 1950. An Introduction to the Theory of Statistics. 14th ed. Griffin, London. 